\title{
Thermophysical modelling for high-resolution digital terrain models
}

\author{
I. Pelivan ${ }^{1,2 \star}$ \\ ${ }^{1}$ DLR Institute of Planetary Research, 12489 Berlin, Germany \\ ${ }^{2}$ Helmholtz Centre Potsdam, GFZ, German Research Center For Geosciences, 14473 Potsdam, Germany
}

Accepted 2018 April 16. Received 2018 March 28; in original form 2017 September 22

\begin{abstract}
A method is presented for efficiently calculating surface temperatures for highly resolved celestial body shapes. A thorough investigation of the necessary conditions leading to reach model convergence shows that the speed of surface temperature convergence depends on factors such as the quality of initial boundary conditions, thermal inertia, illumination conditions, and resolution of the numerical depth grid. The optimization process to shorten the simulation time while increasing or maintaining the accuracy of model results includes the introduction of facet-specific boundary conditions such as pre-computed temperature estimates and pre-evaluated simulation times. The individual facet treatment also allows for assigning other facet-specific properties such as local thermal inertia. The approach outlined in this paper is particularly useful for very detailed digital terrain models in combination with unfavourable illumination conditions such as little-to-no sunlight at all for a period of time as experienced locally on comet 67P/Churyumov-Gerasimenko. Possible science applications include thermal analysis of highly resolved local (landing) sites experiencing seasonal, environment, and lander shadowing. In combination with an appropriate roughness model, the method is very suitable for application to disc-integrated and disc-resolved data. Further applications are seen where the complexity of the task has led to severe shape or thermophysical model simplifications such as in studying surface activity or thermal cracking.
\end{abstract}

Key words: radiative transfer-methods: numerical-comets: general; comets: individual: 67P/Churyumov-Gerasimenko - asteroids: general; asteroids: individual: 25143 Itokawa.

\section{INTRODUCTION}

The utilization of thermophysical models (TPMs) of asteroids and comets supports various applications from scientific data analysis to mission preparation. Thermophysical modelling of e.g. infrared data has proven very useful in determining an asteroid's properties such as size, albedo, and thermal inertia (see e.g. Delbo et al. (2015) and references therein). Knowledge of surface temperatures is important for the design of spacecraft instruments and for the planning of (near) surface operations of missions such as Rosetta with lander Philae and Hayabusa-II with lander Mascot. Yarkovsky and YORP effects, which influence an object's orbital and spin state evolution, can be investigated through thermophysical modelling. The spin-axis orientation and sense of rotation can be constrained by applying TPM techniques such as carried out in Müller et al. (2017). Accurate thermophysical modelling depends on the formulation and applicability (e.g. 1D versus $3 \mathrm{D}$ formulation) of the model as well as the detail of the shape employed. The more detailed the shape for example, the less assumptions have to be made as at least

^E-mail: ivanka.pelivan@gfz-potsdam.de coarse-scale roughness may be resolved by a digital terrain model (DTM).

Thermophysical modelling of asteroids and comets often employs spherical or ellipsoidal shape approximations if the shape has not been determined yet or for simplicity, especially if the shape appears nearly spherical such as the current shape model of Ryugu (see Fig. 1 bottom). For Ryugu, the Hayabusa II target asteroid, Müller et al. (2017) presented several solutions for the preliminary shape and spin axis by matching observational and model data. One solution is favoured (see Fig. 1) based on a careful manual testing. Upon close arrival of the Hayabusa II spacecraft, the accuracy of the model shape will be improved and a refined shape can be derived from imaging data. We do not expect such a surprise as has been the case for 67P/Churyumov-Gerasimenko (CG) - Fig. 1 (top) shows a preliminary model of $\mathrm{CG}$, which has been much altered (see e.g. Fig. 2) upon close encounter of the spacecraft Rosetta. While the simple shapes allow for very quick estimation of surface properties, a closer investigation of surfaces altered by weathering and cratering makes the use of a detailed DTM necessary.

The Hayabusa mission to Itokawa and the Rosetta mission to CG provided a wealth of imaging information that has enabled the construction of very detailed DTMs, see e.g. Preusker et al. (2015) 

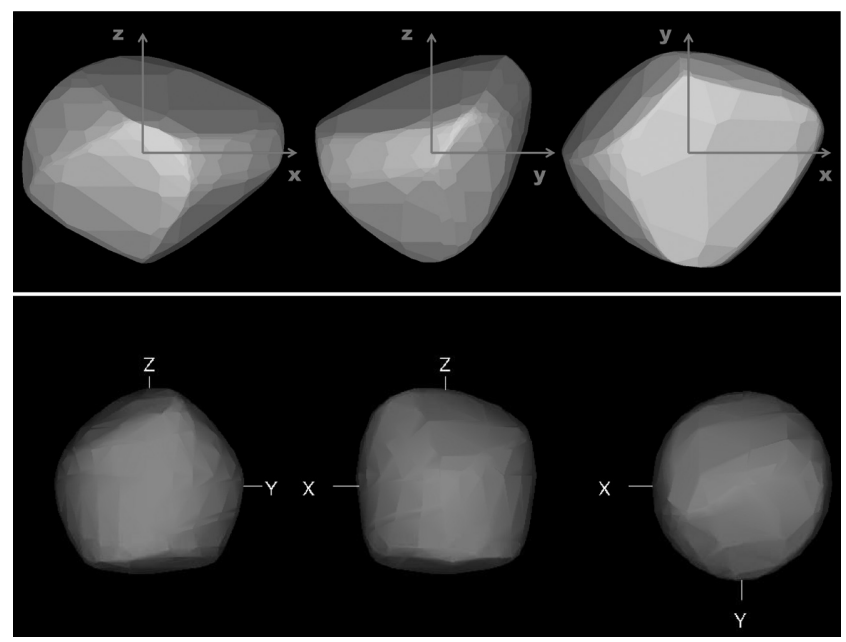

Figure 1. Top: early shape model of CG from Mottola et al. (2014, reproduced with permission (C) ESO), bottom: current Ryugu shape model from Müller et al. (2017, reproduced with permission (C) ESO).
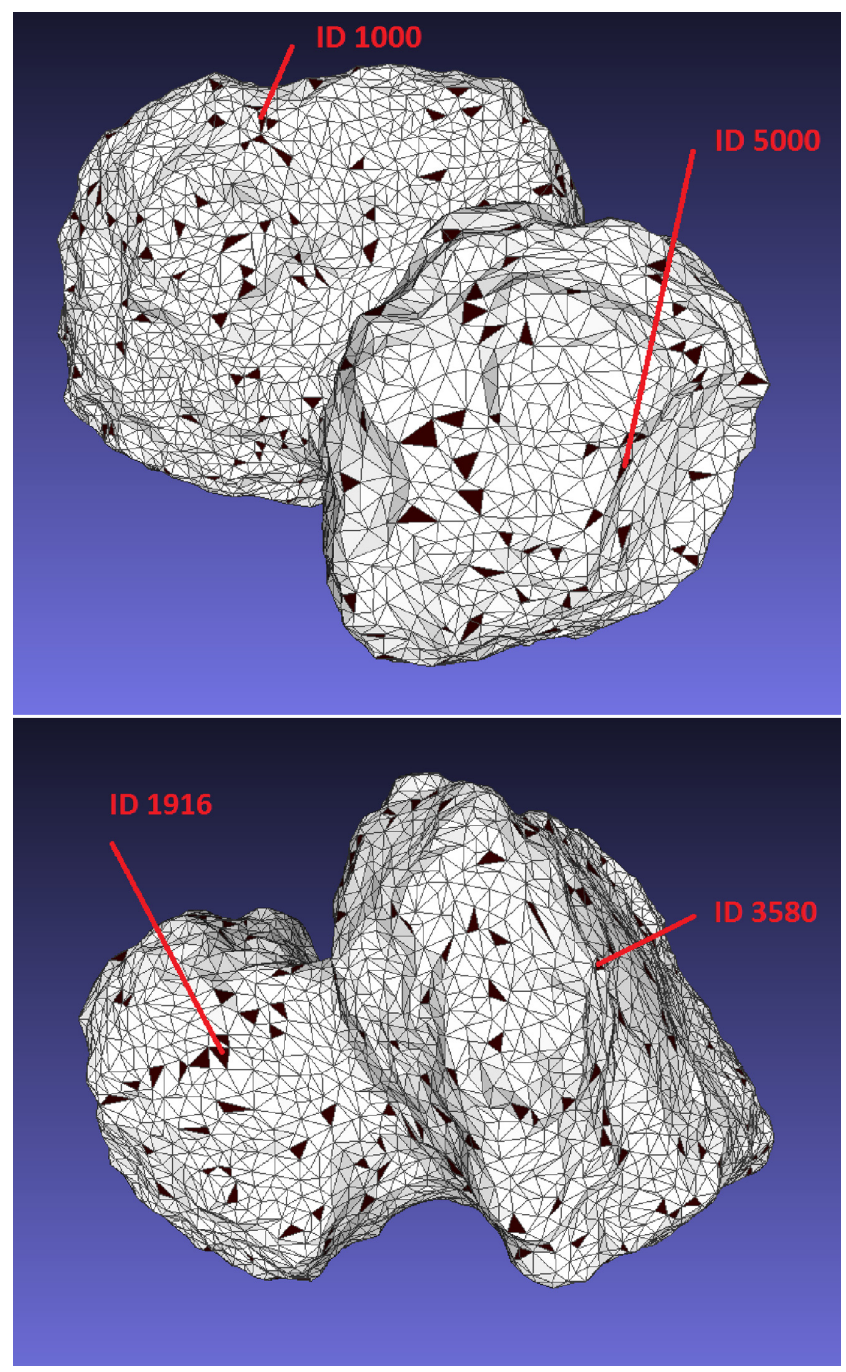

Figure 2. Example DTM of CG with 10000 facets. Elements investigated are coloured in black (semirandom seeding). Results for example facets shown in Figs $4-9$ are labelled. for CG and Gaskell et al. (2008) for Itokawa. For Itokawa, Müller, Hasegawa \& Usui (2014) have applied radiometric techniques to different resolution shape models. Generally, thermophyiscal modelling approaches have not been feasible so far, however, to make use of this detail but a much coarser grid is typically applied for a simulation to run in a reasonable amount of time. The better the local surface (roughness) is modelled in the DTM, the more realistic the thermal modelling becomes. Especially the shape of CG leads to significant self-shadowing and self-heating by thermal radiation from hot surface areas contributing to the energy balance of facing surfaces. The energy budget depends on the thermal conductivity, heating from solar insolation, and infrared self-heating for objects with strong non-convex shapes, and in the cometary case cooling through the latent heat involved in the sublimation process may be present.

The detail of the DTM for the current modelling approach, however, is also limited in using the 1D heat conduction equation, which is applicable if the facet size is large compared to the diurnal skin depth. This typically is the case even for a shape model with millions of facets, which, however, must be checked before application. The utilization of the $1 \mathrm{D}$ heat conduction equation has the big advantage that the computation can be parallelized. Even so, for a high-resolution DTM, the computation per facet may take a lot of time, which is why we seek further optimization. In Section 2 below, the TPM is introduced and the steps undertaken to obtain a fast but accurate solution for surface temperatures and gas production, in the case of active comet modelling, are outlined. The term 'accurate' in this context is used to describe the ability of the method to compute the same results as obtained when utilizing the standard approach that necessitates simulations running up to a few orbits to reach convergence, especially in the case of low surface temperatures. The method has been validated against the standard approach used in Spohn et al. (2015) in obtaining the same temperature curve in a considerably shorter simulation time. The low temperatures derived from measured fluxes emitted from a surface region of Philae's final landing site are associated with a comparatively large $2 \sigma$ uncertainty estimate between +10 and $-25 \mathrm{~K}$ in the worst case for temperatures below $100 \mathrm{~K}$, while for peak temperatures above $120 \mathrm{~K}$, the uncertainty decreases dramatically. This is due to the fact that the instrument has been designed for a much higher temperature range. The model introduced in Section 2 has also been validated successfully against two $3 \mathrm{D}$ implementationsfor a simplified spherical shape (Biele et al. 2015a). Currently, the method is applied to a detailed study of the local landing site environment resolving small-scale roughness. This will enable a direct comparison of model to measured data within the measurement errors. A further application to measured fluxes beyond the one carried out in Spohn et al. (2015) for the purpose of model validation and to test the method's capabilities in producing realistic temperature curves is envisaged for observations of the well-studied asteroid Itokawa. For disc-integrated and generally also for disc-resolved data, model temperatures have to be corrected by a roughness model. Following Rozitis \& Green (2011), roughness may be directly resolved by the method outlined in the following section. A less time-consuming option considers only main energy sources for roughness facets or for the derivation of a correction factor (see e.g. Lagerros (1998) and Davidsson et al. (2015)).

The method introduced in Section 2 is demonstrated in Section 3 for a simplified example shape of the Rosetta target 67P/CG. An application of the method is carried out for high-resolution DTMs of Itokawa and CG in Section 4 to demonstrate the feasibility of thermophysical modelling in combination with large shape models. 


\section{THERMOPHYSICAL MODEL}

\subsection{Basic equations}

For the calculation of surface temperatures, the 1D heat conduction equation is solved for each facet $j$ of a DTM,

$\rho_{j} c_{p, j} \frac{\partial T_{j}\left(x_{j}, t\right)}{\partial t}=\frac{\partial}{\partial x_{j}}\left(\kappa_{j} \frac{\partial T_{j}\left(x_{j}, t\right)}{\partial x_{j}}\right)$.

In equation (1), $\rho_{j}$ is the density, $c_{p, j}$ the specific heat capacity, and $\kappa_{j}$ is the thermal conductivity, $T_{j}\left(x_{j}, t\right)$ is the time- and depth-dependent temperature with $x_{j}$ being the depth variable in the direction of the local surface normal where $x_{j}=0$ is at the surface. Strictly speaking, also the time $t$ is facet-specific as a variable time-step solver is applied; the time-step for output, however, is set constant.

The upper boundary condition is implemented to fulfil the conservation of energy, including effects of local direct and indirect solar irradiation as well as thermal environmental heating,

$U_{j}+W_{j}+u_{j}+w_{j}=\epsilon_{j} \sigma T_{j}^{4}+\kappa_{j} \frac{\mathrm{d} T_{j}}{\mathrm{~d} x_{j}}+Q_{j}$,

where $\epsilon_{j}$ is the thermal emissivity, $\sigma$ is the Stefan-Boltzmann constant, and $Q_{j}$ is the sublimation flux, which is zero for inactive comets or if applied to asteroid thermophysical modelling. Equations (1) and (2) show that local parameters such as the thermal inertia $\Gamma=\sqrt{\rho c_{p} \kappa}$ can vary (see Section 4 below for an application).

The formulations for the direct solar flux $U_{j}$, the diffuse solar radiation $W_{j}$, the direct thermal self-heating $u_{j}$, and the diffuse thermal self-heating $w_{j}$ are given in Davidsson \& Rickman (2014) and Pelivan et al. (2017). They apply for non-convex shapes and can add significantly to the thermal evolution, e.g. in the case of CG (Keller et al. 2015).

For the lower boundary, an adiabatic condition is assumed as detailed in Pelivan et al. (2017). The main differences to the earlier model include a choice of sublimation models based on the standard Hertz-Knudsen formula and the experimental work by Gundlach, Skorov \& Blum (2011) represented by the term $Q_{j}$ in equation (2), whereas the model presented in Pelivan et al. (2017) only allows for application to inactive bodies. Furthermore, while model parameters such as the thermal conductivity could vary with depth, the introduction of local parameter differences has been newly enabled by providing the option to upload parameter maps if available instead of using global variables. This added flexibility makes the current model unique compared to earlier models such as the ones introduced in Lagerros $(1996,1997,1998)$ and Rozitis \& Green (2011), where globally constant parameters are used. It should be noted though that for the purpose of demonstration in Section 3, mostly a constant thermal inertia is applied s.t. equation (1) can be rewritten to locally using a constant thermal inertia value

$\rho_{j} c_{p, j} \frac{\partial T_{j}\left(x_{j}, t\right)}{\partial t}=\kappa_{j} \frac{\partial^{2} T_{j}\left(x_{j}, t\right)}{\partial x_{j}^{2}}$,

which makes the formulation locally equivalent to the earlier model implementations. Regarding the incorporation of DTMs, more flexibility is also added by allowing quadrilateral next to triangular facets.

\subsection{Model implementation}

While the solution to equation (1) with the given upper and lower boundary conditions and starting conditions for intermediate grid points provides depth profiles, the calculation of subsurface temperatures requires a considerable amount of computation time, typically of a couple of orbits to reach convergence. Surface temperatures on the other hand converge rather fast within much less than one orbit given favourable illumination conditions. With appropriate initial conditions, the simulation time can be reduced even further. In the following, subscript $j$ used in equations (1)-(3) to highlight the facet-specific treatment is omitted for better readability; all following equations in this paper, however, refer to individual facets.

Starting conditions for the lower boundary are obtained by calculation of equilibrium temperatures $T_{\text {eq }}$ by averaging estimated surface temperatures $T_{\mathrm{s} \text {, est }}$ over the full orbit. Such derived core temperature is set to $T_{0}=\overline{T_{\mathrm{s} \text {,est }}}$, where $\overline{T_{\mathrm{s} \text {,est }}}$ is the average over all facets and orbit positions, which is valid for constant thermal conductivity (McKay, Squyres \& Reynolds 1986). To obtain starting surface temperatures for all facets, the heat flux in the upper boundary condition is linearized

$U=\epsilon \sigma T_{\mathrm{s}, \mathrm{est}}^{4}+\kappa \frac{T_{\mathrm{s}, \mathrm{est}}-T_{\Delta x}}{\Delta x}+Q$,

where $T_{\Delta x}$ is a starting temperature at depth $\Delta x, \Delta x \geq \delta_{\text {skin }}$ and the skin depth (see e.g. Wesselink 1948) is defined through the thermal conductivity, density, specific heat capacity, and the orbital period $P_{\text {orbit }}$,

$\delta_{\text {skin }}=\sqrt{\frac{\kappa P_{\text {orbit }}}{\pi \rho c_{p}}}$.

Equation (4) is iterated until the right-hand side equals the solar flux. At the start of this iteration, the initial difference between the right-hand side and the solar flux is used to reset the starting $T_{\Delta x}$, thereby reducing the number of iteration steps. Even without the latter measure, the iterative process to obtain starting conditions is very fast and only takes minutes on a standard PC for a model with 10000 facets.

The estimated surface temperatures are used directly for each facet as starting conditions at a given point in orbit while setting the lower boundary to $T_{0}=\overline{T_{\mathrm{s}, \text { est }}}$ as explained above. In doing so, convergence times for surface temperatures are reduced considerably (see Section 3 below) to a fraction of the orbit, resulting in a few days to a couple of weeks of simulated time (depending on illumination conditions). Note that for the calculation of $T_{0}$ alone, the heat flux term in equation (4) can be omitted.

When using a temperature-dependent thermal conductivity, equation (4) is modified:

$U=\epsilon \sigma T_{\mathrm{s}, \mathrm{est}}^{4}+f(\kappa) \frac{T_{\mathrm{s}, \mathrm{est}}-T_{\Delta x}}{\Delta x}+Q$,

where $f(\kappa)$ depends on the nature of $\kappa(T)$, i.e. for a simple temperature dependency, $f(\kappa)=\kappa(T)$; otherwise, a linearized function is inserted. Also, instead of using a global lower boundary condition, a facet-specific boundary condition $T_{0}=T_{0, j}=\overline{T_{\mathrm{s}, j \text {,est }}}$ is applied, where $\overline{T_{\mathrm{s}, j}, \text { est }}$ is an average over time for each facet.

When including rough surfaces by solving the $1 \mathrm{D}$ heat conduction equation for each roughness facet of a topographical model corresponding to the approach outlined in Rozitis \& Green (2011), best starting conditions are obtained when also considering diffuse solar radiation $W$ and direct thermal self-heating $u$ on the left-hand side of equation (4). 
Table 1. Example objects investigated. References provided refer to the shape model used. References for the thermal inertia are given at the beginning of Section 3 .

\begin{tabular}{llccl}
\hline Case & Object & Facets & $\Gamma\left(\mathrm{Jm}^{-2} \mathrm{~K}^{-1} \mathrm{~s}^{-1 / 2}\right)$ & Reference \\
\hline 1 & CG & 10000 & $30-300$ & Preusker et al. (2017) \\
2 & CG & 125000 & 30 & Preusker et al. (2017) \\
3 & CG & 1500000 & 30 & Preusker et al. (2017) \\
4 & Itokawa & 49152 & 700 & Gaskell et al. (2008) \\
5 & Itokawa & 200000 & $200-1200$ & Gaskell et al. (2008) \\
6 & Itokawa & 3145728 & 700 & Gaskell et al. (2008) \\
7 & Ryugu & 1144 & 200 & Müller et al. (2017) \\
\hline
\end{tabular}

\section{DEMONSTRATION OF METHOD}

The following investigation has been carried out for different celestial objects listed in Table 1 varying in shape and model resolution, and for different orbit positions as well as thermal inertia. While representing examples for testing purpose, the thermal inertia values incorporated are based on the low values derived in Gulkis et al. (2015) for CG. Due to locally higher values possible (Spohn et al. 2015) and to widen the test range, higher values are also applied in the comet case. For the asteroid examples, thermal inertia values are based on Müller et al. (2014) and references therein under the assumption of rough surfaces for Itokawa, and Müller et al. (2017) for Ryugu (best-fitting results were obtained for low-to-no surface roughness). The thermal inertia in the current investigation is treated as a model parameter; therefore, underlying model assumptions in the derivation of the thermal inertia are not critical.

The findings outlined in the following are of general nature. For illustration purpose of the method, mostly a 10000 facet model of CG is used and results for an orbit position of 3 au are shown. The example model is a reduced shape model from a follow-up DTM of Preusker et al. (2015). Cases 1, 4, and 7 of Table1 are not considered high-resolution DTMs, but are used to test the method before applying it to the more time-consuming actual high-resolution cases. Application to Ryugu is carried out to prepare for a possible application to the high-resolution model expected from near-surface imaging upon close encounter of the Hayabusa II spacecraft mid2018 such as carried out in Takita, Senshu \& Tanaka (2017), but possibly focusing more on highly resolved local areas of the asteroid.

Calculations have been performed for a sublimating comet as this yields lower surface temperatures with typically longer simulation times necessary. The method, of course, is as valid for the inactive case.

In order to verify that the calculated temperatures are converged, for a representative sample of surface elements, long simulation runs are performed such that the dependence on initial boundary conditions is lost. For all sample facets marked black in Fig. 2, multipleday and three-orbit simulations have been carried out, the three-orbit simulations representing the converged case. For a smaller subset of elements, long simulation runs of 20 orbits have been performed to prove that after one to three orbits indeed convergence is reached for all facets. Of this smaller subset, results are presented for four facets (labelled in red in Fig. 2), which are characteristically different in illumination duration and diurnal temperature profile due to the extreme shape of CG and season. For a more convex shape, temperature curves will not be so fundamentally different. The diversity of temperature curves is documented in the literature - a diurnal variation that could have been observed on a spherical body is shown in Capria et al. (2017) (Fig. 13) for a location on the big lobe that experiences similar illumination conditions at the time of

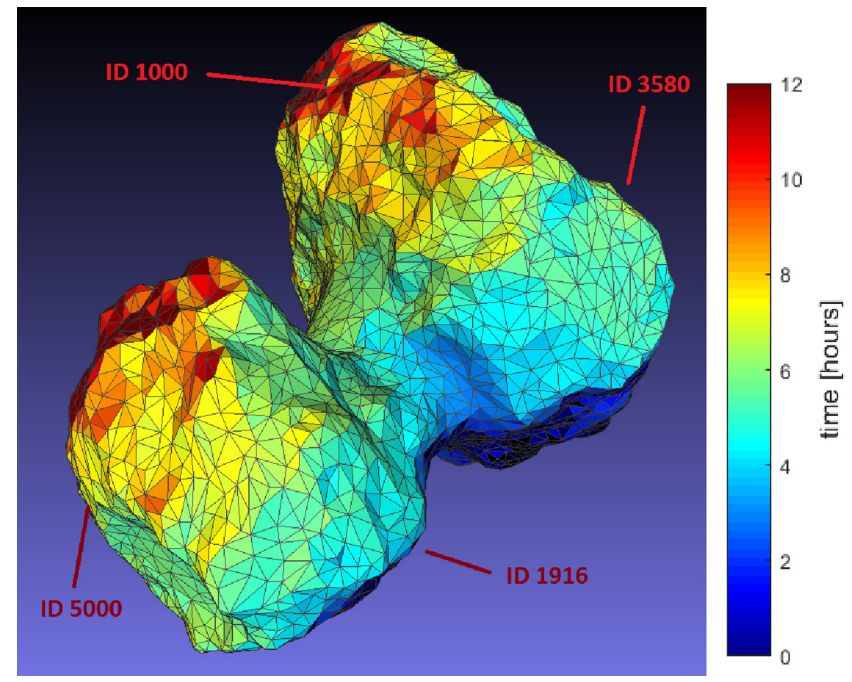

Figure 3. Cumulative hours of sunlight over one comet rotation at 3 au. ID 1000 is displayed; the other example IDs from Fig.2 are approximately marked. Colouring in dark red: fully illuminated over one comet rotation, dark blue: total shadow.

measurement as example ID 5000 on the small lobe (at a different time in orbit). Due to environmental shadowing, the measured temperatures at the local landing site of Philae on CG are quite different (Spohn et al. 2015). From data presented in Schloerb et al. (2015), while showing millimetre and submillimetre temperatures for effective latitudes, it can be inferred that surface temperature curves vary in showing a broad peak such as calculated for example ID 1000 due to a long illumination time down to narrower peaks at smaller effective latitudes. While these data points cannot be directly compared to the current model calculations due to different observation times, looking at these measurements allows to judge that model results are realistic.

For demonstration purpose, a low thermal inertia of $\Gamma=30 \mathrm{Jm}^{-2} \mathrm{~K}^{-1} \mathrm{~s}^{-1 / 2}$ is applied in the simulation. The fastness of convergence is highly dependent on thermal inertia - the smaller it is, the longer the simulation time necessary; see also equation (11) of Kuehrt (1984). The example presented therefore represents the worst case in terms of thermal inertia of all configurations investigated. Indeed, all other models from Table 1 converge much faster with and without optimized initial conditions, but all configurations benefit from improved starting conditions.

Fig. 3 shows the cumulative hours of sunlight over one comet rotation at the orbital position of $3 \mathrm{au}$. The example facets with numbers 1000, 1916, 3580, and 5000, chosen for demonstration purpose, are indicated (for exact locations, see Fig. 2). Example facet 1916 is in permanent shadow at this position, while the other facets are sunlit for a varying amount of time.

Surface temperatures depend on a good resolution of the subsurface grid to accurately resolve the vertical temperature gradient. We therefore first test grid independence of the method applied to exclude errors introduced by a poorly resolved depth profile. The grid is set up in the following way:

$$
\begin{aligned}
x_{i} & =x_{i-1}+\Delta_{x}, \quad i=0 \ldots N \\
\Delta_{x} & =s_{\text {grid }}^{i} \Delta_{x 1} \\
\Delta_{x 1} & =\frac{b-a}{t_{\text {grid }}}, \quad a=0 \quad \text { (surface) } \\
t_{\text {grid }} & =\sum_{i=0}^{N} s_{\text {grid }}^{i} .
\end{aligned}
$$




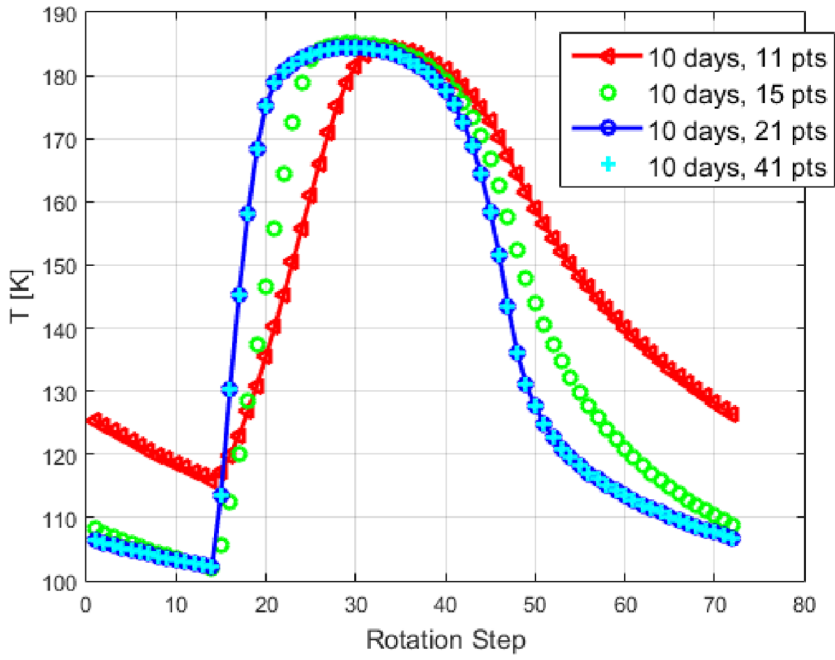

Figure 4. Influence of grid on CG surface temperatures exemplified for facet 5000. Each rotation step shown corresponds to a body rotation of 5 deg

In equation (7), $s_{\text {grid }}$ is a free parameter (for the current study, a value of 1.2 has been used to obtain a dense grid near the surface) and $N$ is the number of grid points, varied from 10 to 100 , and $b$ extends to several skin depths. The grid evaluation is only shown for one of the chosen example facets labelled in Fig. 2 since the same trend is observed for all facets of the DTM. As can be seen in Fig. 4, a grid with around 20 points is necessary when using the non-linear spacing of equation (7) to set up the numerical domain in depth to exclude grid-spacing effects that negatively affect computation time.

In Fig. 5 and particularly in Fig. 6, the advantage of using precomputed initial surface temperatures is demonstrated. Fig. 5 shows surface temperatures for one comet rotation for two facets that are illuminated for at least a moderate amount of time or at an angle that leads to a comparatively high peak temperature. The simulation time to reach convergence is short - less than $20 \mathrm{~d}$, corresponding to less than 40 comet rotations in this case, with arbitrary starting conditions.

In Fig. 6, computed surface temperatures for two facets are shown that experience extreme conditions in terms of solar illumination: The top figure shows the results for a facet that is completely shadowed at the given position in orbit over one rotation period, while the second facet is illuminated for about $2 \mathrm{~h}$ only, corresponding to less than a sixth of the comet day.

For all facets in Figs 5 and 6, results for different simulation times and starting conditions are compared. The simulated results after a run time of 3 and 20 orbits match. This is considered the converged solution. Furthermore, two simulations are shown where a simulation time of $10 \mathrm{~d}$ is used before results are output at $3 \mathrm{au}$. In one case, an initial upper boundary condition of $100 \mathrm{~K}$ (equal to the lower boundary condition) is applied, the same starting condition as used for the 3 and 20 orbit simulations for which arbitrary surface starting conditions lose influence. In the other case, the temperature iterated by applying equation (4) $10 \mathrm{~d}$ ahead of reaching the orbit position of 3 au is used. For facets that are sunlit for at least a moderate amount of time or at an angle that leads to a comparatively high peak temperature, a simulation time between a couple of days and a few weeks suffices for convergence. For less favourable illumination conditions, the simulation time increases: a simulation time of $20-50 \mathrm{~d}$ is typically necessary when pre-computed starting
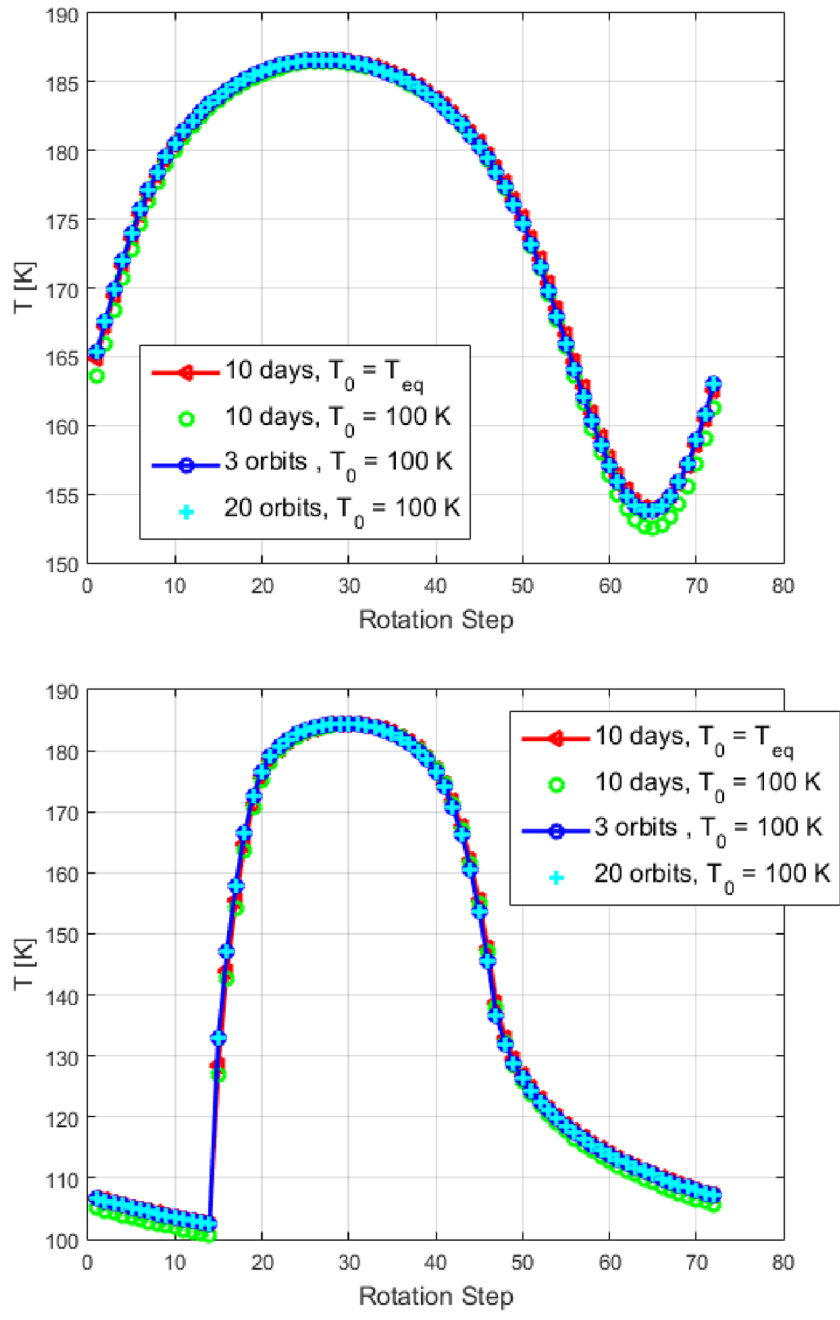

Figure 5. Two CG facet temperatures. Top: facet 1000 in near-permanent sunlight and bottom: facet 5000 with a moderate duration of insolation.

conditions are applied. Fig. 7 shows for comparison how long it might take for less illuminated facets to obtain a converged solution if starting conditions are not optimized. Using the comet core temperature as starting condition for all facets, for shadowed and mostly shadowed facets even half an orbit of computation time is not sufficient to obtain a converged diurnal surface temperature evolution. It takes about one orbit for the solution to converge in the shadowed case. With that knowledge, it is possible to assign each facet not only a starting temperature, but also a starting time, i.e. when to start the simulation to obtain a converged solution at the desired output time. While uncommon, this reduces the overall simulation time considerably. For the current example utilizing a very low thermal inertia, low-temperature facets are assigned starting times 100-200 d ahead of the output time, while moderately lit facets are assigned starting times of 50-100 d and facets illuminated for a long time have starting times of $12-50 \mathrm{~d}$ with corresponding initial temperature conditions. A higher thermal inertia leads to faster convergence - for the above example, using a thermal inertia of $\Gamma=300 \mathrm{Jm}^{-2} \mathrm{~K}^{-1} \mathrm{~s}^{-1 / 2}$, the surface temperature of the shadowed facet converges after a fourth of the time necessary for the low thermal inertia case presented above.

Next to the facet-specific starting temperature and the more unusual facet-specific simulation time based on the initial condition 

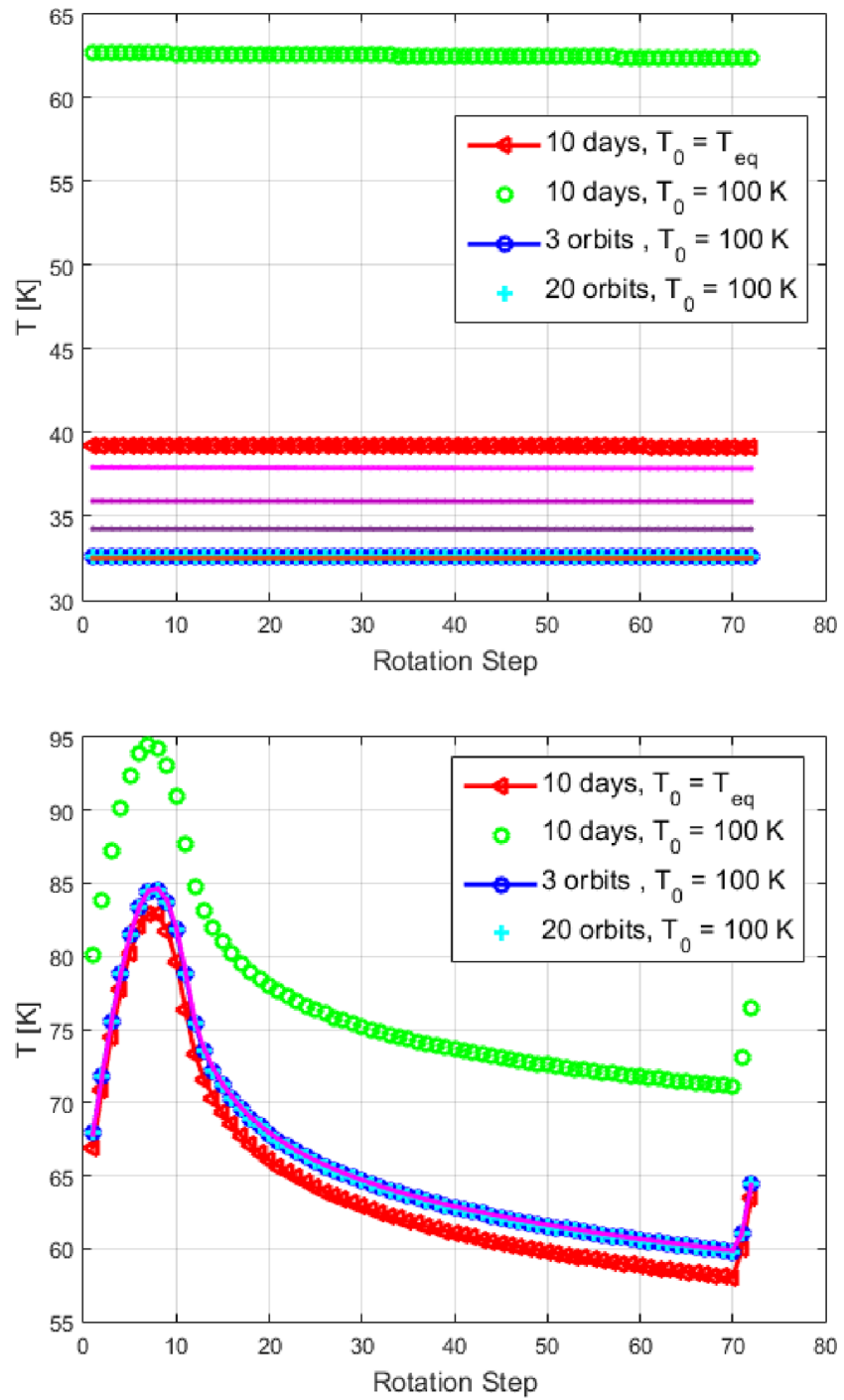

Figure 6. Two CG facet temperatures, top: facet 1916 in permanent shadow and bottom: facet 3580 with a short duration of insolation. Lines without legends: top: increasing number of simulation time $(20,50,100,200 \mathrm{~d})$ with decreasing solution value, bottom: $20 \mathrm{~d}$ of simulation time is sufficient in this case for a converged solution when using starting conditions iterated $20 \mathrm{~d}$ ahead of the output time.

calculated in advance, other facet-specific properties can also help to speed up the overall computation time. A good example is the introduction of a facet-specific thermal inertia or thermal conductivity $\kappa=\kappa_{i}$ for facet $i$. Using a model that has implemented a global thermal inertia, several simulation runs are necessary to piece together regions with different thermal inertia values. A model that on the other hand allows to incorporate facet-specific physical properties that can be read in e.g. as a properties map of the DTM has the advantage to cover different model regions within a single run. An example is shown in Section 4 below for a DTM of Itokawa.

All of the investigations above have used a constant thermal inertia based on constant thermal conductivity, specific heat capacity, and density. The temperature dependence of the thermal conductivity has been investigated in the framework of the KOSI experiments (see e.g. Seiferlin et al. 1996) and more recently by Gundlach et al. (2011). Combining millimetre and submillimetre thermal flux data, Gulkis et al. (2012) show that a model with a single constant ther-
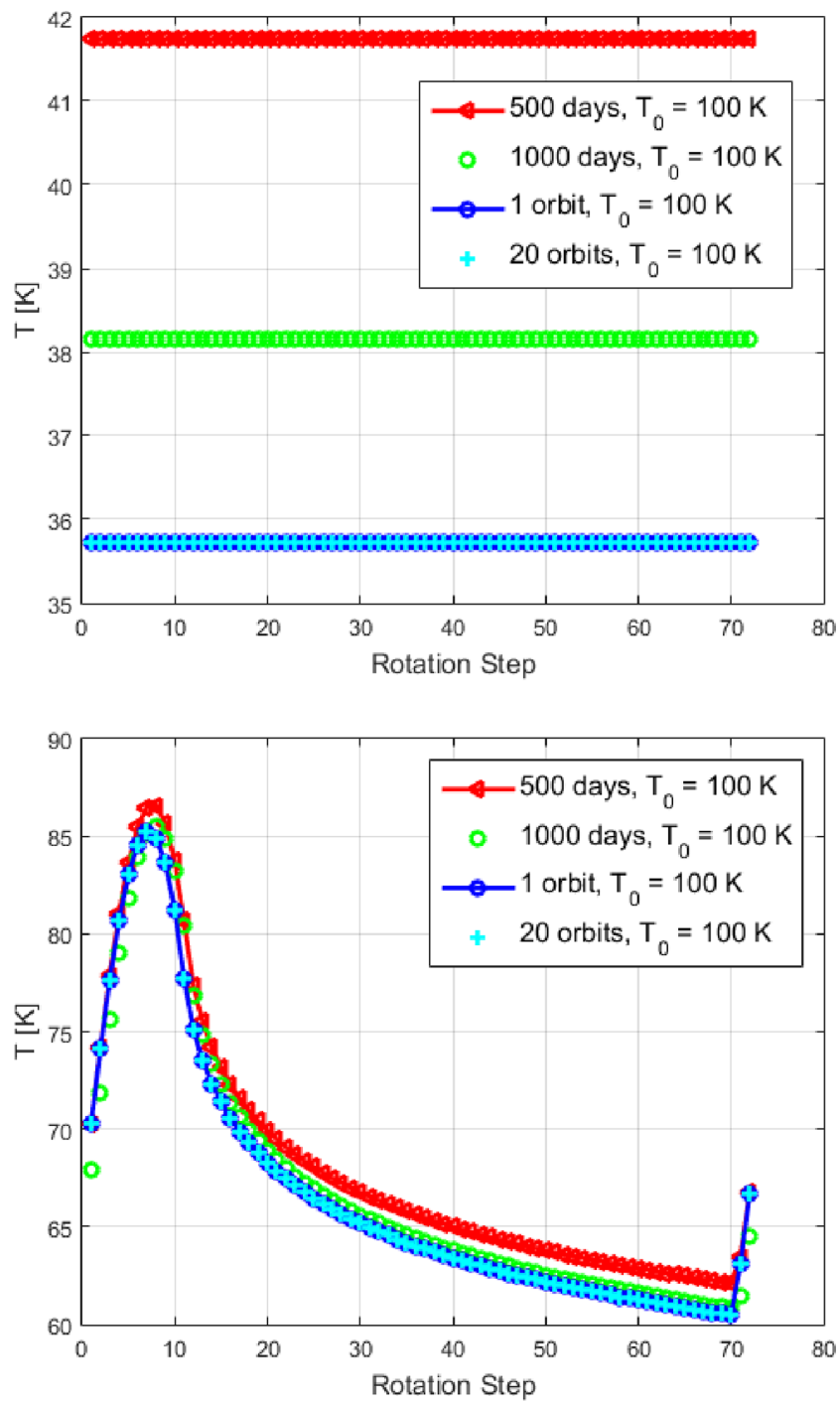

Figure 7. CG facet temperatures using the core temperature as starting condition, different simulation times. Top: facet 1916 and bottom: facet 3580 .

mal inertia cannot be used to fit both measurements. In order to test if the method presented in this paper is of advantage when using a temperature-dependent thermal inertia as well, several models for the thermal conductivity, including that of Gundlach \& Blum (2012), have been evaluated with varying model parameters. For the purpose of demonstration, the comparatively simple formulation of Steiner, Kömle \& Kührt (1991) is used, in which an effective temperature-dependent thermal conductivity is defined as

$\kappa_{\text {eff }}=\kappa+\kappa_{p}$,

where $\kappa_{p}$ is a 'pore conductivity' depending on a pore radius, the gas mass emission rate, the latent heat of sublimation, and a structural material parameter depending on the volume fraction covered by the pores (see Steiner et al. 1991 for further details). Model parameters in equation (8) are adjusted such that the effective thermal conductivity is equal to the constant value used before at a peak temperature of $T=185 \mathrm{~K}$. Therefore, by construction, the difference in high-temperature facets is not as pronounced as for those in the low-temperature regime. In Fig. 8, the converged solutions of two example facets shown in Figs 5 and 6 are compared to results 

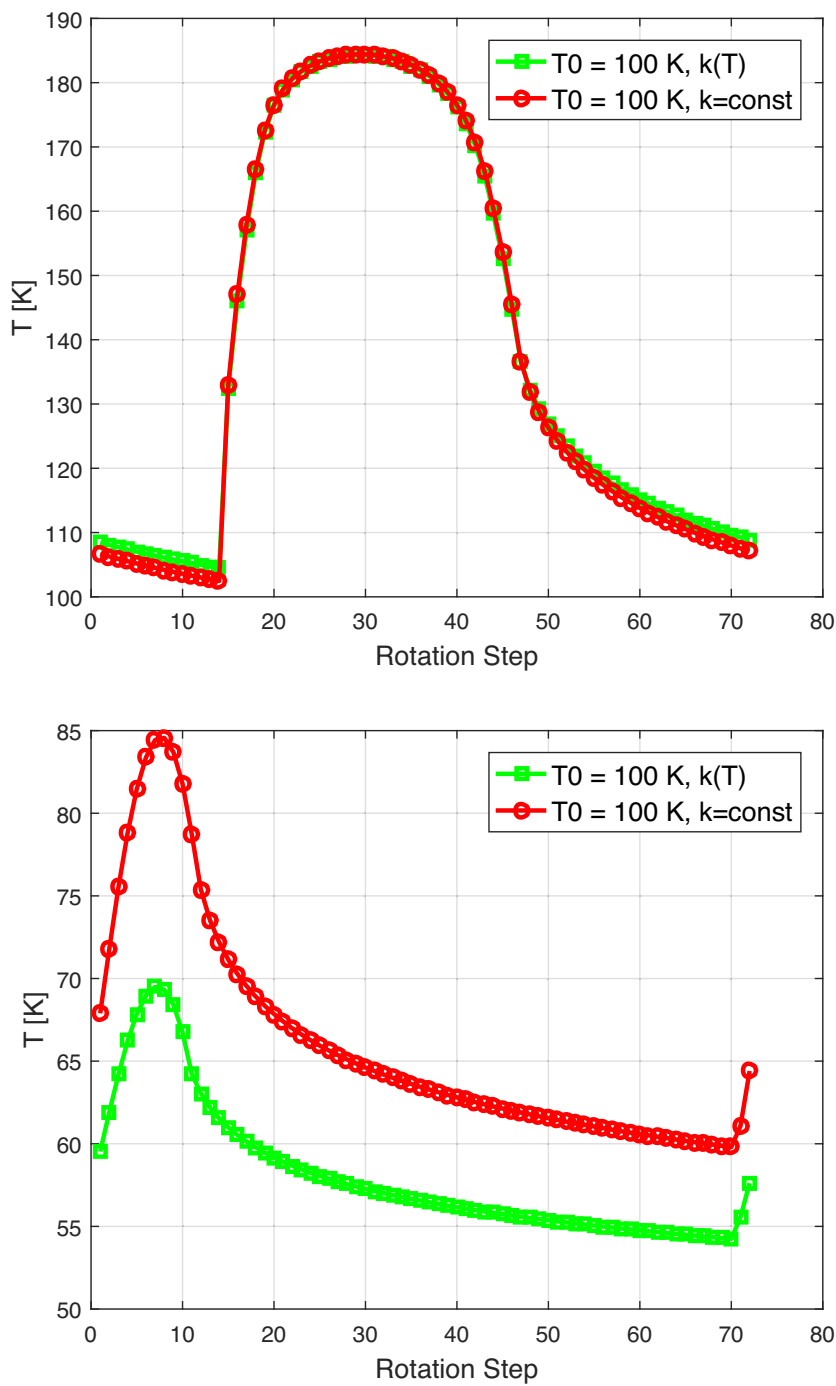

Figure 8. CG facet temperatures using the core temperature as starting condition; comparison between constant $(\mathrm{k}=\mathrm{const})$ and temperature-dependent thermal conductivity $(\mathrm{k}(\mathrm{T}))$ : top: facet 5000 and bottom: facet 3580 .

using the same simulation parameters except for a replacement of the constant thermal conductivity by equation (8). Due to the applied temperature dependency of the thermal conductivity, surface temperatures for example facet 3580 are lower than before. In this comparison, the simulation is run with arbitrary starting conditions until convergence has been reached. To ensure this, a 3-orbit run has been compared to a 20-orbit run, delivering the same results.

In Fig. 9, model runs are repeated using newly derived starting conditions for the temperature-dependent thermal inertia case. The temperature dependence of the thermal conductivity slows down convergence for low-temperature facets, probably because in this case the overall temperature is lowered, but pre-estimated surface temperatures still have a large effect. For the lower boundary, instead of deriving the core temperature following McKay et al. (1986) in calculating a common core temperature for all facets, when using a temperature-dependent thermal conductivity, depth temperatures have to be evaluated differently. Good results are reached when averaging facet temperatures individually over the orbit instead of applying a global core temperature. In Fig. 9, it can be seen that simulated surface temperatures match the converged case (top of
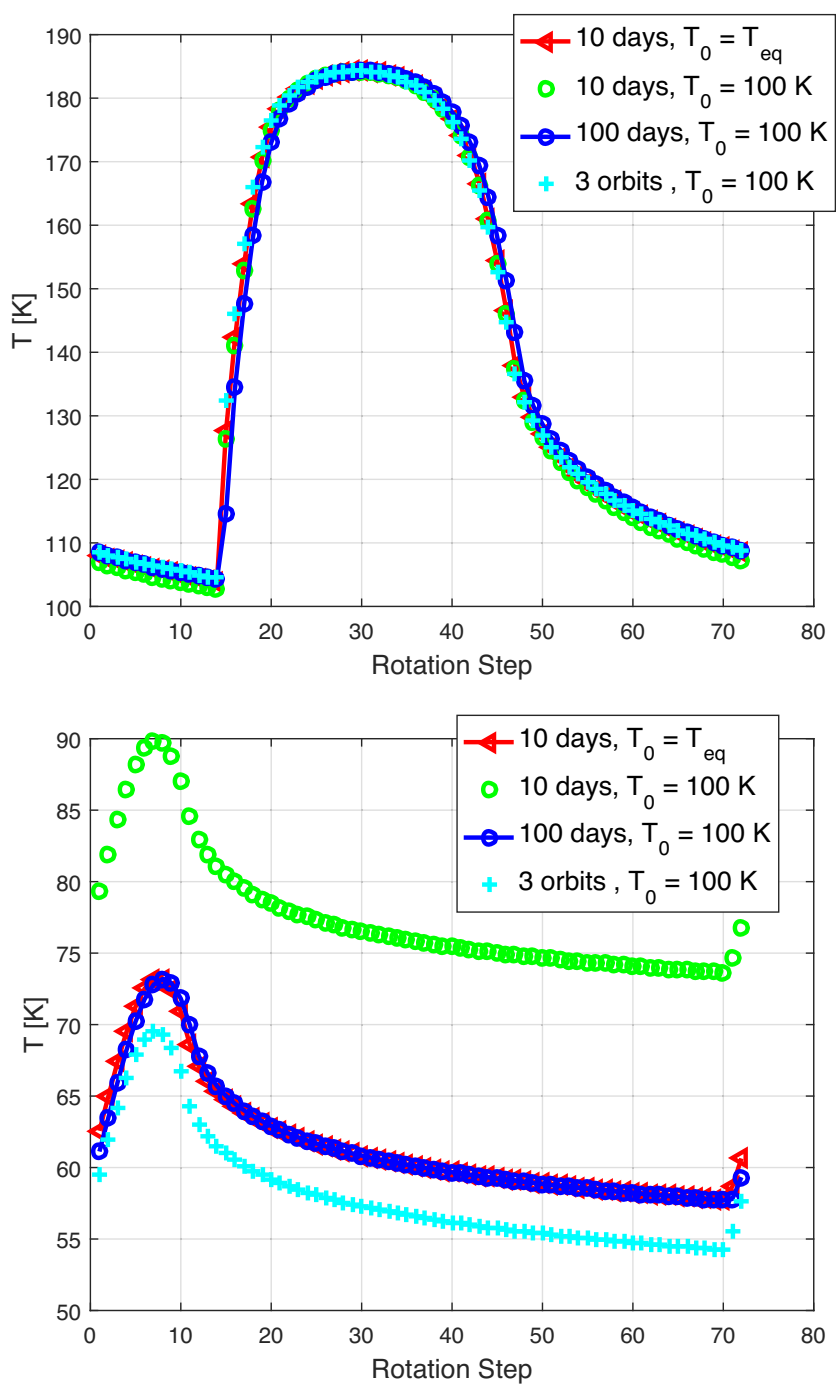

Figure 9. $\mathrm{CG}$ facet temperatures with different starting conditions; temperature-dependent thermal conductivity $(\mathrm{k}(\mathrm{T}))$, top: facet 5000 and bottom: facet 3580 .

figure) or are nearing convergence (bottom) after $10 \mathrm{~d}$ with improved starting conditions. Without pre-iterated starting conditions, it takes about 10 times more to obtain the same intermediate results. For example ID 5000, $100 \mathrm{~d}$ of simulation without optimized starting conditions initially results in an improvement; however, the temperature curve then deviates from the converged solution, indicating that a longer run is necessary for an overall match in this case.

We have not included surface roughness or other features such as layering in this model investigation. Surface roughness can be applied as correction to computed surface temperatures such as done in Müller et al. (2014) based on the models by Lagerros (1996, 1998). When using the approach based on the beaming function described in Lagerros (1998), the method outlined above is not affected whether it is used with or without surface roughness. As shown by e.g. O'Rourke et al. (2012), adding surface roughness is necessary to explain thermal measurements. As already mentioned, when using the method presented in this paper for the evaluation of disc-integrated and disc-resolved data, it must be complemented by a roughness model. Incorporating other model features such as lay- 


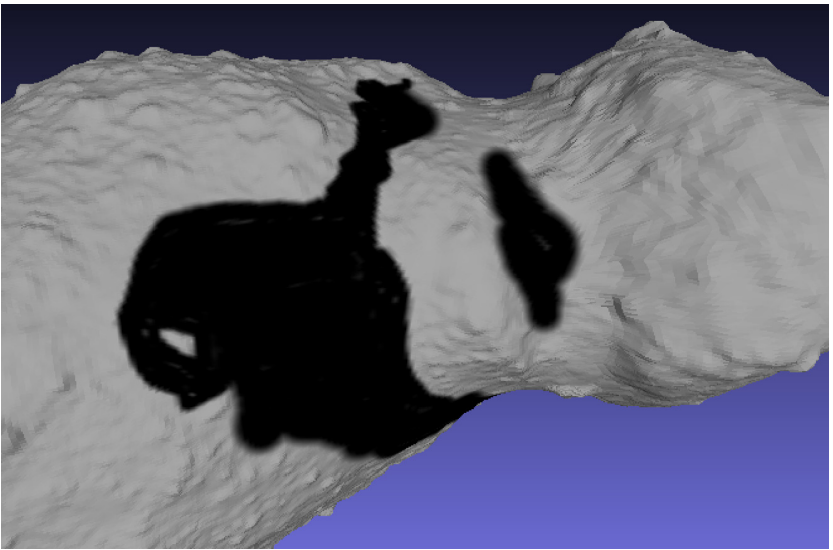

Figure 10. Itokawa shape model with facet-specific thermal inertia ( 200 000 facets), coloured in black: thermal inertia of $\Gamma=500 \mathrm{Jm}^{-2} \mathrm{~K}^{-1} \mathrm{~s}^{-1 / 2}$; all other regions: $\Gamma=900 \mathrm{Jm}^{-2} \mathrm{~K}^{-1} \mathrm{~s}^{-1 / 2}$.

ering, also a different iteration scheme to obtain starting conditions is utilized, including the different layer boundaries. The overall method as presented above, however, remains the same.

\section{MODEL APPLICATION}

In this section, several model applications targeting two celestial bodies are shown, demonstrating the method's versatility for scientific and operational purpose. In Section 4.1, the Itokawa shape models are employed. In Section 4.2, the focus is on applications to $\mathrm{CG}$.

\subsection{Application to Itokawa}

Fig. 10 shows the Itokawa 200000 facet model divided into two thermal inertia regions, roughly following the terrain identified by Demura et al. (2006) and Miyamoto et al. (2007). The coarsely constructed thermal inertia map marks facets with a thermal inertia of $\Gamma=500 \mathrm{Jm}^{-2} \mathrm{~K}^{-1} \mathrm{~s}^{-1 / 2}$ in black; all other regions are assigned a thermal inertia of $\Gamma=900 \mathrm{Jm}^{-2} \mathrm{~K}^{-1} \mathrm{~s}^{-1 / 2}$, according to the findings of Müller et al. (2014) based on modifying the roughness level in their TPM applied to disc-integrated infrared measurements of Itokawa. While the degeneracy between roughness and thermal inertia has to be kept in mind, this model application could be a starting point for a re-analysis of observational data taking into account locally different thermal inertia values and applying a local roughness model as well.

In computing facet temperatures, a core temperature of $\sim 180 \mathrm{~K}$ has been evaluated as described in Section 2.2 above. In Fig. 11, results of two simulations are displayed. The top image shows the temperatures for a rotation instant of 2005 November 19, using a uniform thermal inertia of $\Gamma=700 \mathrm{Jm}^{-2} \mathrm{~K}^{-1} \mathrm{~s}^{-1 / 2}$, while for the bottom figure the facet-specific thermal inertia of the two regions shown in Fig. 10 are applied. The colouring in Fig. 11 is scaled to the maximum temperature of $357 \mathrm{~K}$ for the rotation instant. Contrasting the two models, the largest differences can be seen at the regions with a thermal inertia of $\Gamma=500 \mathrm{Jm}^{-2} \mathrm{~K}^{-1} \mathrm{~s}^{-1 / 2}$ when comparing to the uniform case. The lower thermal inertia leads to significantly lower temperatures during nighttime, while scaled daytime temperature differences are not that apparent for the date investigated.

The thermophysical simulations are repeated for Itokawa's lowresolution model of $\sim 50000$ facets as well as the highest res-
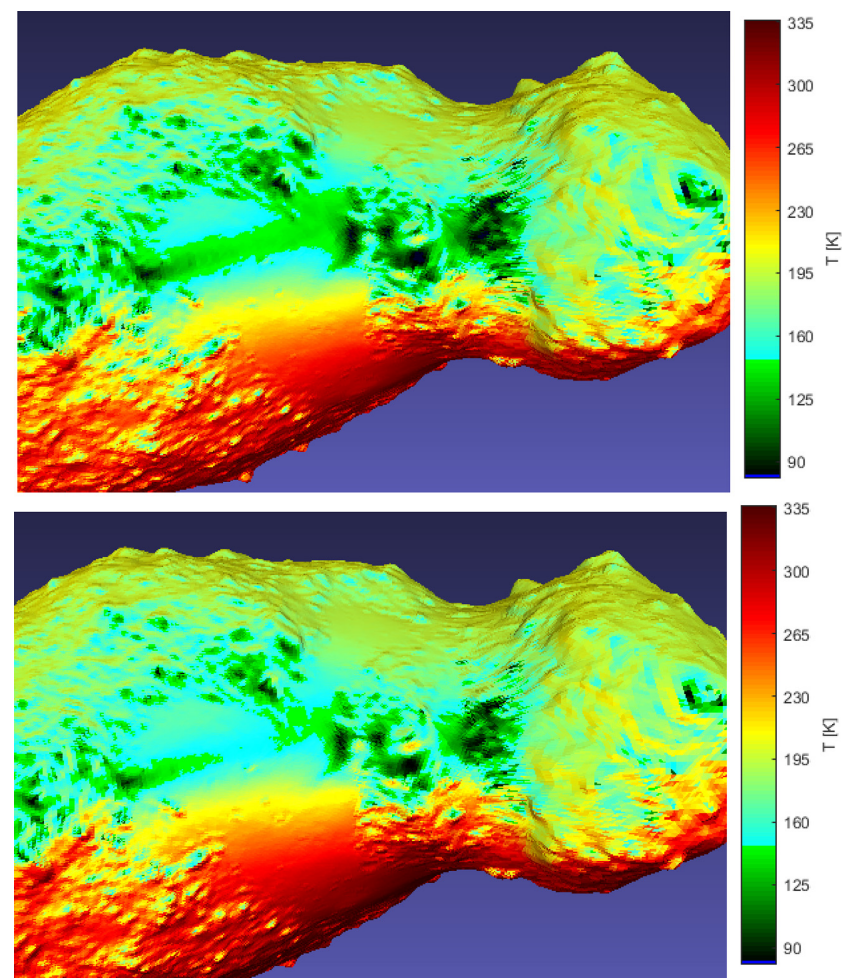

Figure 11. TPM results for Itokawa during 2005 November 19. Top: uniform thermal inertia of $\Gamma=700 \mathrm{Jm}^{-2} \mathrm{~K}^{-1} \mathrm{~s}^{-1 / 2}$ and bottom: thermal inertia map of Fig.10. The colouring is scaled to the maximum temperature of $357 \mathrm{~K}$ for the rotation instant shown.

olution model with over 3 million facets. A thermal inertia of $\Gamma=700 \mathrm{Jm}^{-2} \mathrm{~K}^{-1} \mathrm{~s}^{-1 / 2}$ is applied to all facets.

In Fig. 12, surface temperatures around noon are shown for a part of the smaller lobe for the two Itokawa shape models with the lowest and highest resolution. When comparing the low-resolution model to the DTM with the highest resolution, the same temperature distribution can be observed on a large scale. The coarse model's flattening effect, however, leads to the disappearance of hot spots that show in the lower centre of the depicted area in the bottom of Fig.12. From this comparison alone, it becomes clear that a roughness model is needed when using the low-resolution DTM for analysis. Investigating the influence of surface roughness in a thermal inertia optimization process, Müller et al. (2014) report that even for the highest Itokawa shape resolution, a surface roughness model is necessary to explain the observed infrared fluxes.

Further quite pronounced differences in using a detailed versus less detailed DTM show in the temperatures themselves. Highest temperatures for the region shown in Fig. 12, displayed in red, amount to just over $315 \mathrm{~K}$, while lowest are just above $93 \mathrm{~K}$ (detailed model with over 3 million facets). For the low-resolution model of just below 50000 facets, the highest/lowest temperatures calculated for that region are around $285 / 120 \mathrm{~K}$. This clearly also is an effect of shape resolution in smoothing out detail in the 50k model, while the high-resolution DTM resolves at least coarse-scale roughness.

\subsection{Application to CG}

The desired model accuracy depends on the usage - e.g. for the investigation if a lander or its parts will survive low temperatures on an atmosphereless body, knowledge of accurate low temperatures is of importance (see Pelivan et al. 2017). If the activity of a comet is of 


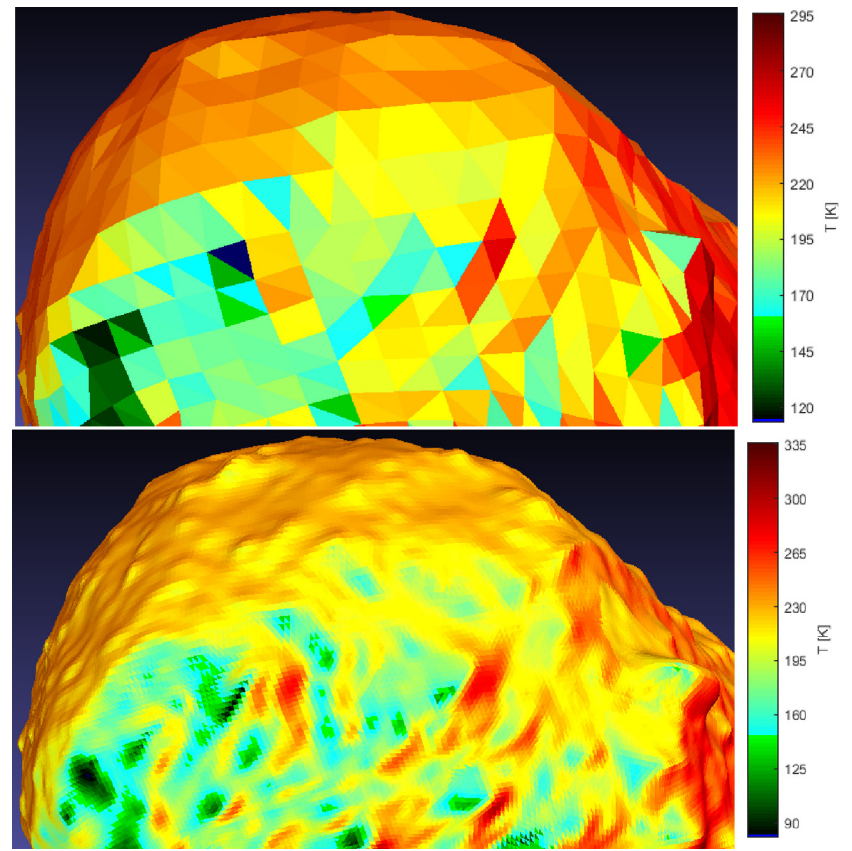

Figure 12. Surface temperature on Itokawa small lobe on 2005 November 19, around noon, top: Itokawa shape model with 49152 facets and bottom: Itokawa shape model with 3145728 facets. Red/blue facets: hot/low temperatures colour-scaled to highest (individual) model temperatures.

concern, low temperatures that do not contribute to gas production do not need to be modelled precisely and thus the simulation time can be considerably shortened since low-temperature facets require the most calculation time.

Even with the capabilities to study a very detailed DTM, it may not be desirable to do so. Generating data for a specific date is considered the typical application. When investigating the temperature evolution and derived quantities along the full or longer parts of the orbit, a smaller model not considerably affecting accuracy is preferable to a full-resolution DTM. The current method can aid here in timely checking for a few sample points along the orbit, which reduction in facet number still gives acceptable results. In Fig. 13, results for 2 of 15 sample days in CG's orbit are shown, where the cumulative water production (water is used as single sublimating species in this run) for a fully active comet is calculated for DTM number 2 of Table 1. A fully active comet here means that each facet is sublimating across its full area. The 125000 facet DTM is downsized into smaller models, the smallest merely having 1500 facets. Fig. 14 shows the relative difference in water gas production between the largest DTM and those reduced in size. While the relative difference increases with heliocentric distance, the overall gas production drastically decreases towards aphelion. Investigation of e.g. the smallest shape model with merely 1500 facets reveals a maximum relative deviation in water gas production of around 2 percent at perihel, around 4 percent at a heliocentric distance of $3 \mathrm{au}$, and of about 14 percent at aphelion. Water production at $3 \mathrm{au}$, however, is an order of magnitude smaller than at perihelion and negligible (four orders of magnitude smaller) at aphelion. The comparison in Figs 13 and 14 shows that a DTM with 12500 facets, i.e. 10 per cent of the original size, is sufficient for calculation of the diurnal gas production of the complete surface (at aphelion, the maximum difference in gas production is around 4 per cent for this shape model).
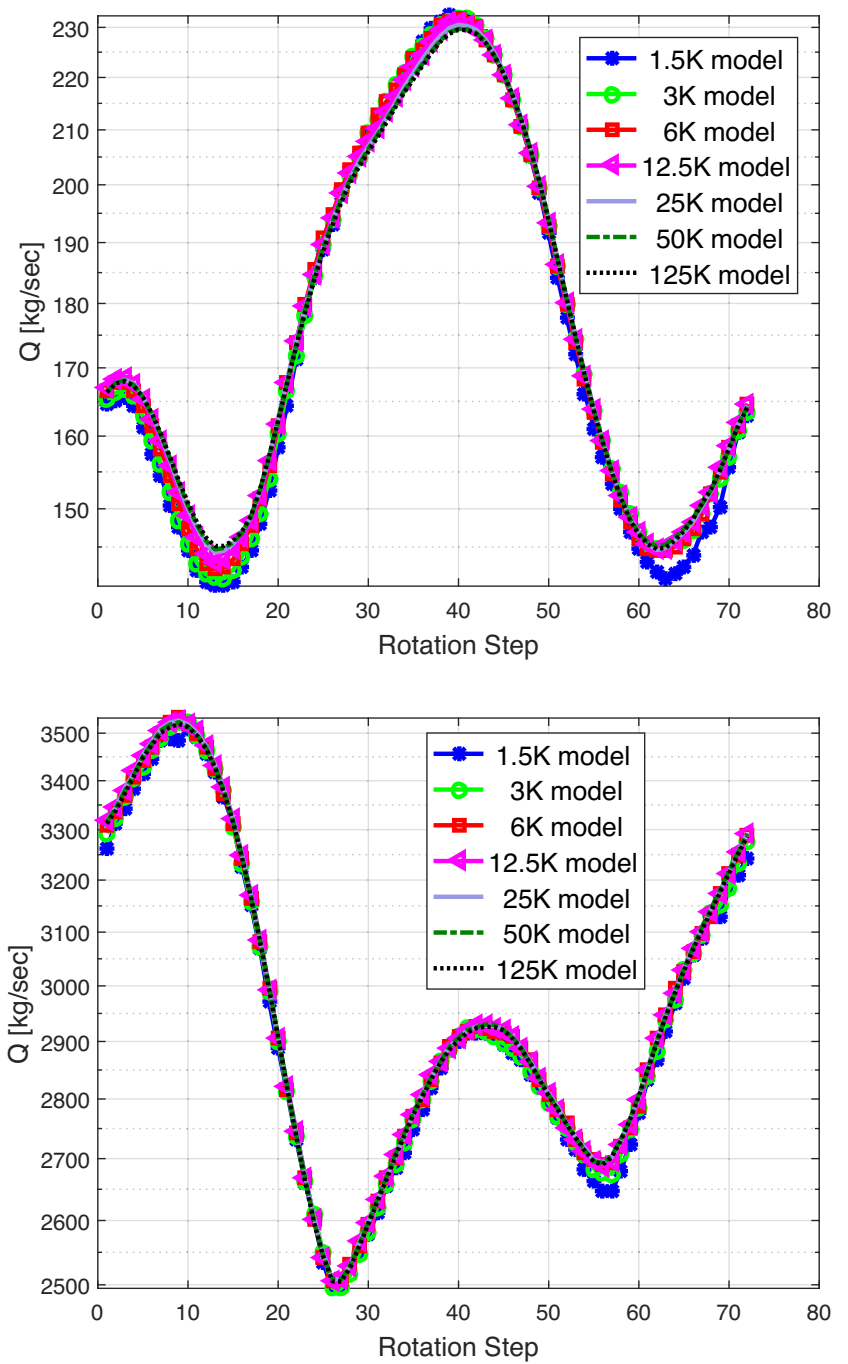

Figure 13. Diurnal water production for downscaled DTMs of CG, different points in orbit. Top: around 3 au and bottom: at perihel.

In Fig. 15, CG's water gas production using a simplified thermal model is compared to results using the physically more representative model presented in Section 2. The simplified model is represented by evaluating the upper boundary condition neglecting or linearizing heat conduction, as it is often performed, see e.g. Keller et al. (2015). In the current approach, equation (4) is applied for comparison. As it otherwise is used as a good initial guess for starting conditions, it did not warrant adding an iterative approach to include self-heating. Therefore, the top of Fig. 15 shows the comparison for both models neglecting self-heating, while in the bottom the simplified model without self-heating is compared to the full model with self-heating. The top figure shows that the simplified model overestimates gas production due to slightly overestimated peak temperatures. Coincidentally, the simplified model results without any added physics match better when comparing with the full thermal model including self-heating. The question arises if it makes sense to include self-heating in a simplified model if the model uncertainty amounts to the same order of magnitude as the self-heating effect.

The last application targets a case where the accuracy of low surface temperatures is of importance. Instead of landing in a wellilluminated and obstacle-free place, lander Philae of the Rosetta 

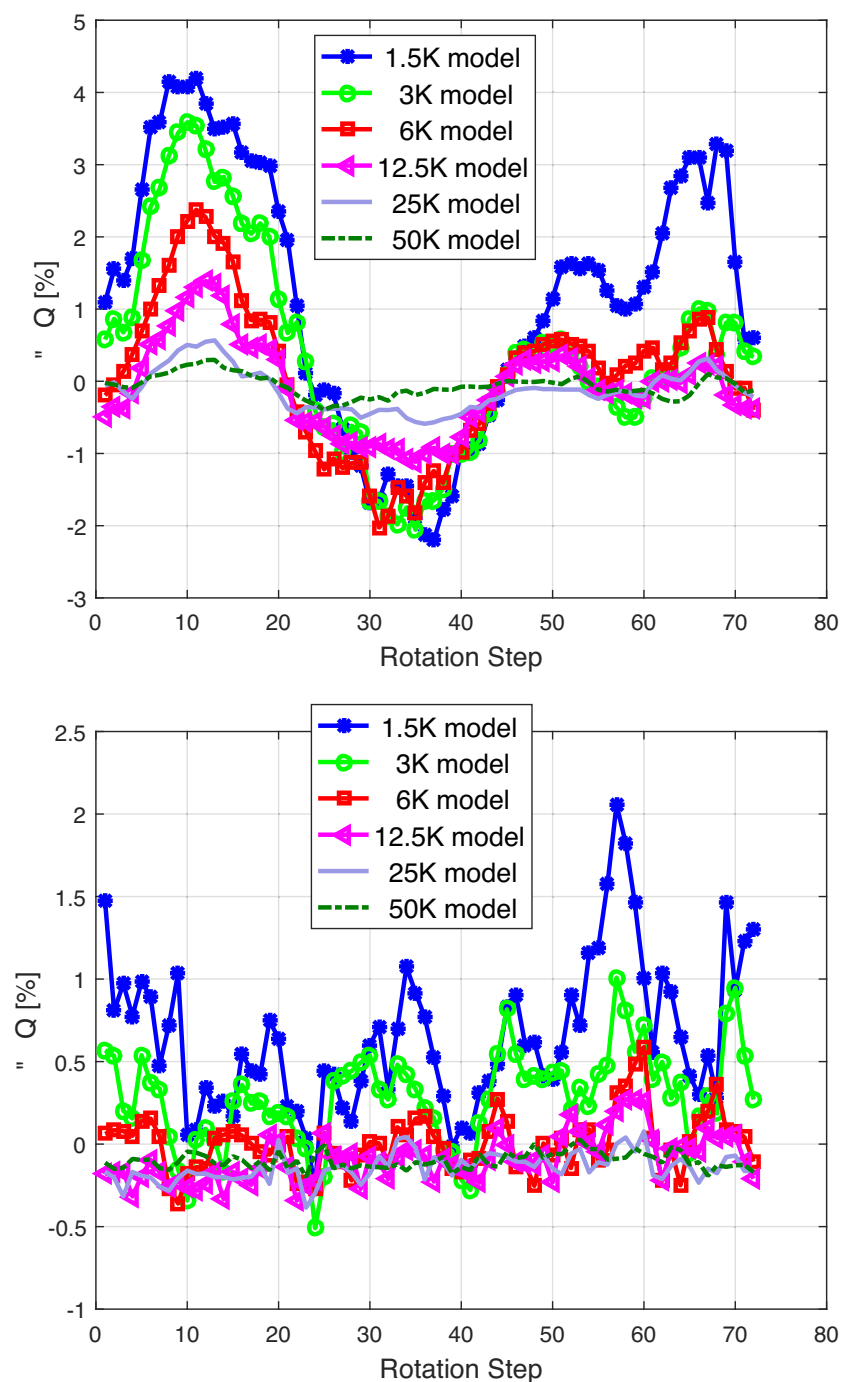

Figure 14. Difference in water production between largest and downscaled DTMs of CG, different points in orbit. Top: around 3 au and bottom: at perihel.

mission to CG bounced off and finally settled in a very different environment with very short illumination periods (Biele et al. 2015b).

The MUPUS (Multipurpose Sensors for Surface and Sub-Surface Science) instrument package was designed to gain information on surface and subsurface properties of the local landing site. MUPUS consists of three instruments, of which the thermal mapper (TM), an infrared radiometer located on the balcony of the lander, measured data that allowed a first derivation of the local temperature at the landing site upon artificially introducing blockage of daylight (Spohn et al. 2015).

The exact landing position has not been known for a very long time and the lander orientation is still under discussion. Based on imaging data, a preliminary environmental model (see Fig. 16) could be constructed based on a global large-scale DTM, the local Abydos region (Capanna et al. 2015), and ROLIS and CIVA instrument data (see Ulamec et al. 2016 for an overview of lander instruments and Kömle et al. 2017 for a description of the preliminary shape model). For this preliminary DTM, exact surface temperatures are calculated for all facets in the TM's field of view (FOV), taking into account self-heating from surrounding facets. Fig.17 shows
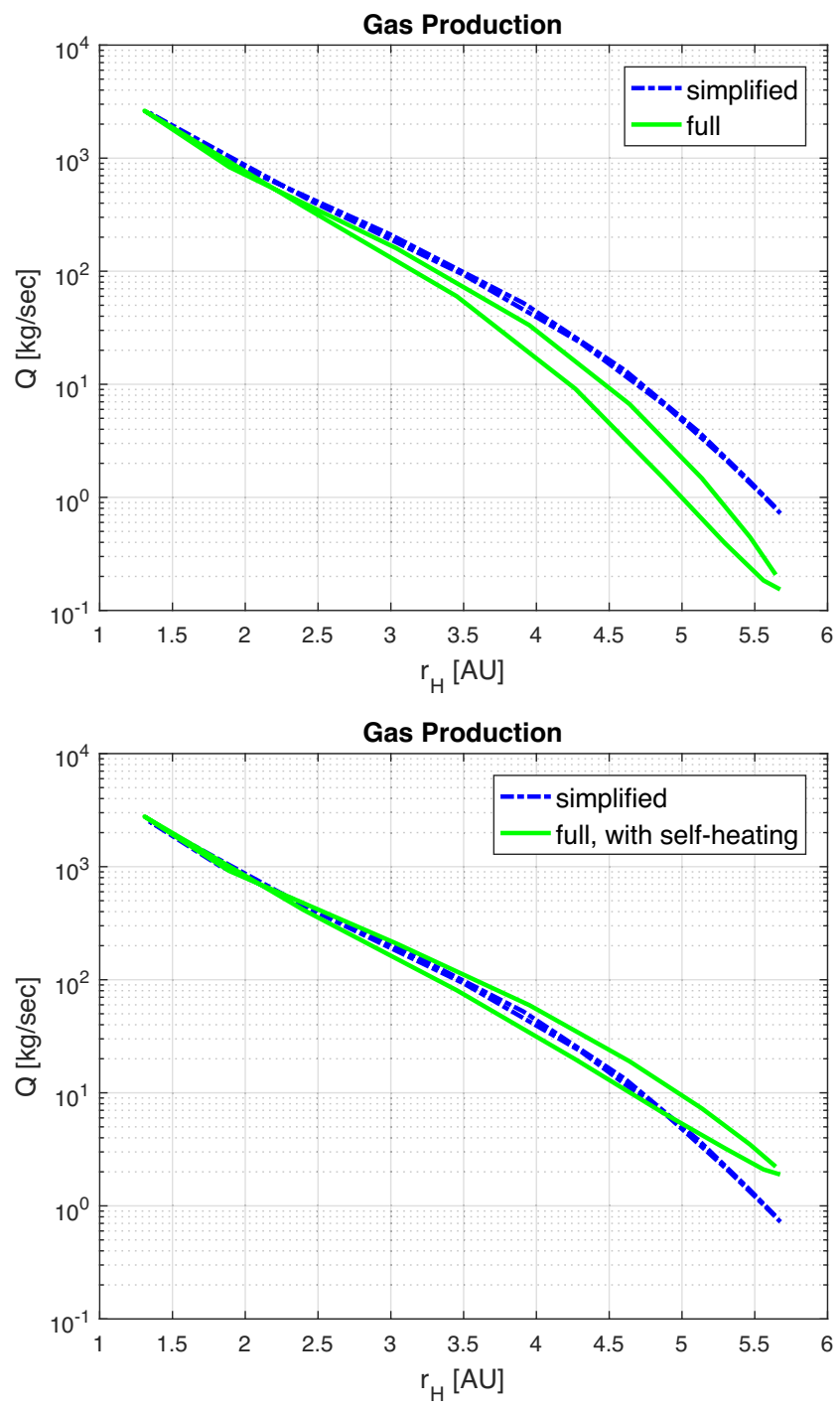

Figure 15. Water production rates versus heliocentric distance - simplified: evaluated with equation (4), full: using the full thermal model with (bottom) and without (top) self-heating.

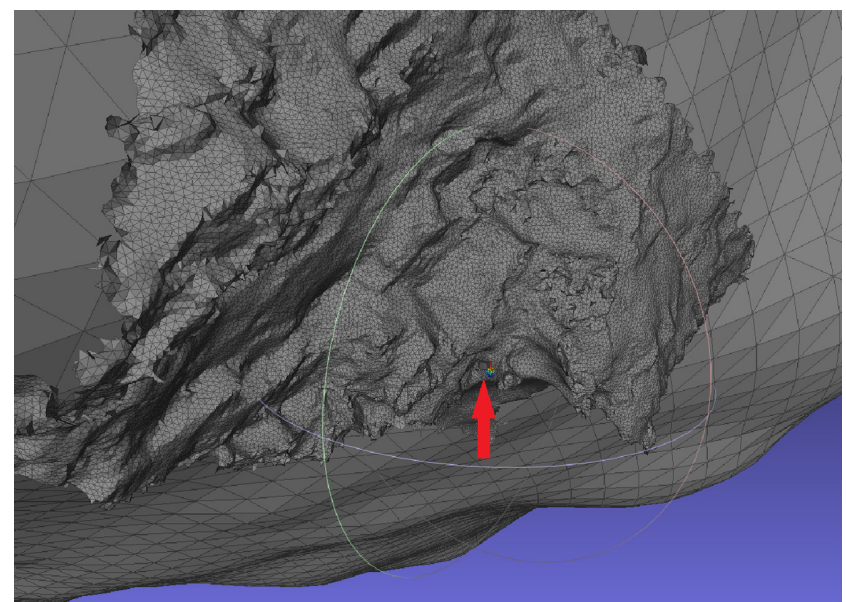

Figure 16. Preliminary Abydos local shape model - reconstruction of Abydos (Capanna et al. 2015) combined with global shape model of CG. The arrow shows the location of lander Philae. 


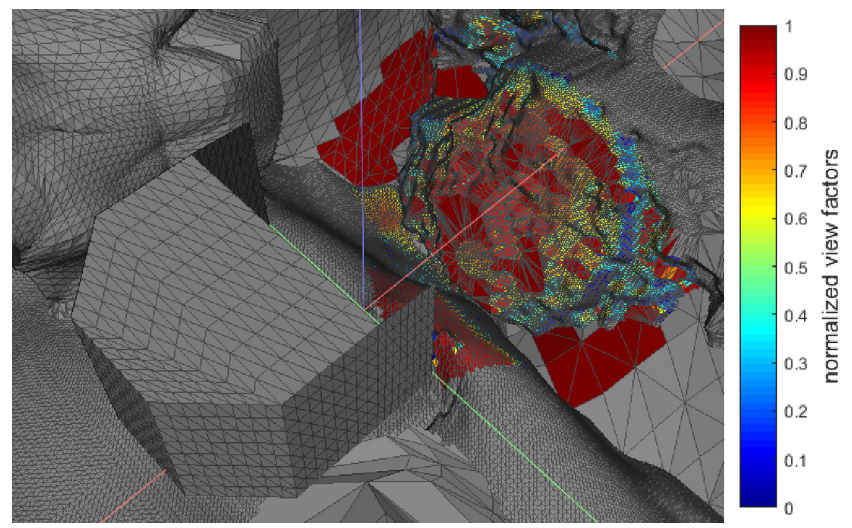

Figure 17. MUPUS-TM instrument view factors within local landing environment.

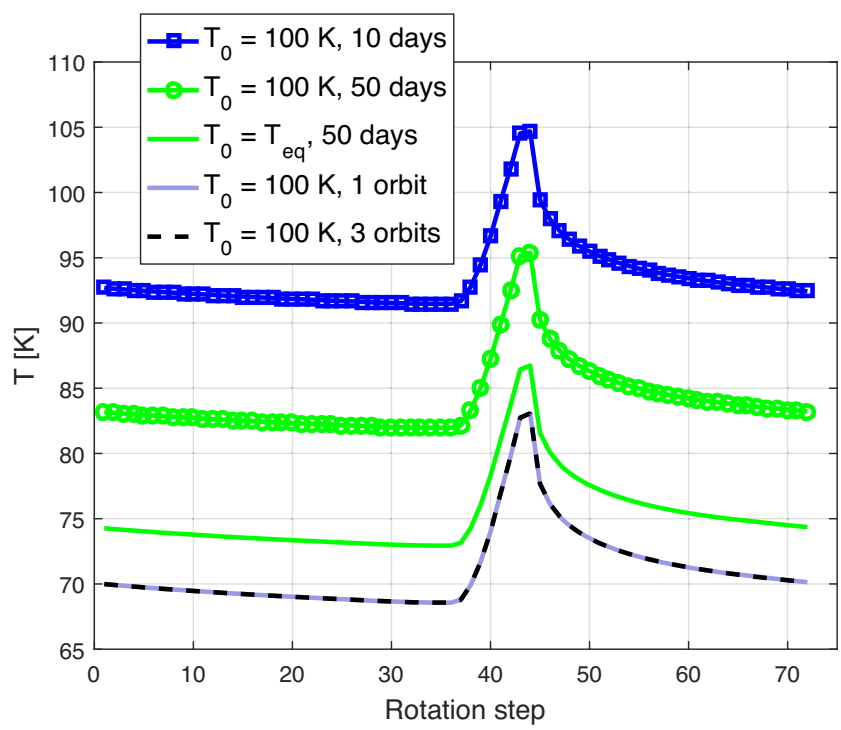

Figure 18. Diurnal temperature variation for example facet in TM's FOV at 3 au. Thermal inertia: $\Gamma=67 \mathrm{Jm}^{-2} \mathrm{~K}^{-1} \mathrm{~s}^{-1 / 2}$.

all facets considered (coloured), i.e. all facets that are in the TM's FOV. The colouring scheme demonstrates the relative importance of the single facets in contributing to the overall flux sensed by the TM, where red shows maximum and blue minimum weight. The weight of the single facets is determined by the angle between the facet normal and the TM direction, the distance to the instrument and the size of the facet. Outer facets gain importance if they are comparatively large. This effect is lessened by the TM sensitivity roughly following a Gauss distribution. The instrument sensitivity is applied to the final temperatures computed with the thermal model.

In Fig. 18, the diurnal surface temperature evolution for an example facet of the TM's FOV is shown. A thermal inertia of $\Gamma=67 \mathrm{Jm}^{-2} \mathrm{~K}^{-1} \mathrm{~s}^{-1 / 2}$ is applied, which is within the range of $\Gamma=85 \pm 35 \mathrm{Jm}^{-2} \mathrm{~K}^{-1} \mathrm{~s}^{-1 / 2}$ found by Spohn et al. (2015) where the local thermal inertia at the landing site has been determined as comparatively high compared to the global range of $\Gamma=10-50 \mathrm{Jm}^{-2} \mathrm{~K}^{-1} \mathrm{~s}^{-1 / 2}$ for the subsurface layers as inferred in Gulkis et al. (2015). Indications for a locally sintered near-surface micro-porous dust-ice layer point towards higher subsurface thermal inertia, which is in contrast to the findings of Gulkis et al. (2015).
However, while the less-resolved global findings are thought to be representative for larger areas of the comet, locally there may be large deviations from the global value as detected at CG's final landing site.

Due to an instrument failure to penetrate the subsurface layers, the depth- and temperature-dependency of the local layering remain unknown. Therefore, in this first refinement study, facet thermal inertia is assumed to be constant. The results for simulation durations of $10 \mathrm{~d}$ (without optimized starting conditions) are compared to those of $50 \mathrm{~d}$ (with and without optimized starting conditions), one orbit and three orbits. It can clearly be seen that a few comet rotations of simulation time are not sufficient to obtain a converged solution. Increasing the simulation time improves the results, especially when optimized starting conditions are used. Most facets in the TM's FOV require simulation times above 50 up to $200 \mathrm{~d}$, when using appropriate initial conditions. Above a couple of $100 \mathrm{~d}$, the starting conditions begin to lose importance unless the initial guess is rather far off

The limiting factor for a final in-depth TM data analysis lies in the still prevailing uncertainties for the detailed local landing environment and the final lander orientation. The preliminary shape model used in this example does not reflect the correct illumination duration on the lander itself. The model facets in the TM's FOV are cumulatively illuminated for about the measured amount of time if not the correct time spans, pointing to the wrong lander or individual facet orientation for the preliminary DTM used.

\section{CONCLUSIONS}

Highly resolved celestial body shapes are computationally very demanding when performing a thorough thermophysical investigation. This is especially true for highly non-convex shapes by itself or in combination with disc-integrated data. Also highly resolved local landing sites on nearly convex shapes such as expected to be obtained for Ryugu will benefit from a model that can take into account a local DTM with large detail. A method is presented for efficiently calculating converged surface temperatures and temperature-dependent quantities. While the equations applied solve for subsurface temperatures as well, the method is only applicable to surface temperatures. The software itself can be used to calculate depth profiles as well, losing the method's advantage, however, of a reduction in computation time. The only speed-enhancing measure in that case is the implemented parallelization.

The method presented adds several pre-processing steps to the actual thermophysical modelling. Some calculation steps, however, are fast - the iterative evaluation of the upper boundary condition to obtain starting conditions takes seconds to minutes depending on the size of the shape model. The calculation of illumination conditions is not fast, but is already required as input to the evaluation of the upper boundary condition of the TPM. The illumination duration drawn on in combination with estimated surface temperatures to set facet-specific simulation times is a by-product of the data already available. The full method is feasible for a single calculation to obtain data for a specific date, which is the typical application, e.g. for mission planning purpose or to aid in data analysis. Automation to e.g. calculate temperatures along orbit steps is not very straightforward unless accuracy of low-temperature facets can be neglected.

The present investigation shows that the speed of surface temperature convergence depends on many factors, which include the quality of initial boundary conditions, thermal inertia, illumination 
conditions, and resolution of the numerical depth grid. For temperature convergence with arbitrary initial conditions and low thermal inertia, a model run over hundreds of days may be required if the initial guess is far off, especially if illumination conditions lead to low surface temperatures. Depending on the application, more or less accuracy may be required. For the investigation of near- and mid-infrared fluxes that are dominated by high-temperature facets, the accuracy of the low-temperature facets may not be that critical. The same holds true for the study of activity on a comet where low-temperature facets do not contribute at all. For the investigation of a poorly lit landing site as happened in the case of Philae on CG, the accuracy of low-temperature facets is highly important. The thermal modelling approach presented in this paper therefore is particularly useful for the application to detailed DTMs in combination with unfavourable illumination conditions such as occurring in craters, caused by cliffs and overhangs and general rough areas.

For accurate TPM predictions applied to real data, a more realistic handling of physical properties has to be carried out. Thermal inertia shows a depth dependency (see e.g. Keihm et al. 2012), a dependency on heliocentric distance (Marsset et al. 2017), as well as on the diurnal cycle (Delbo et al. (2015) and references therein) and rotation rate (Harris \& Drube 2016), all indicative of a changing thermal inertia as a function of temperature and possibly due to layering-induced changes of physical properties. A small study suggests that the method works also well for temperature-dependent thermal conductivity. For any application to measurements, this aspect has to be investigated more thoroughly.

We have presented two applications to DTMs of Itokawa and CG to show the applicability of the method and to exemplify its usefulness. For Itokawa, it is demonstrated how to make use of the model's facet-specific properties implementation by applying a facet-specific thermal inertia, next to estimated boundary conditions and simulation time. A comparison of two vastly different DTMs with respect to resolution shows the suppression of hot spots on the surface that are resolved in the high-resolution DTM, and the reduction of temperature range in the low-resolution DTM.

For CG, a study of DTM size reduction has been conducted to determine the lowest acceptable model size for a further detailed investigation of activity evolution along the orbit assuming a fully active comet. Convergence of low-temperature facets not contributing to activity has not been ensured in favour of computation speed. A case where the exactness of low surface temperatures matters is represented by the unplanned final landing site of Philae, the lander of the Rosetta mission. It is illustrated that optimized starting conditions can improve model results towards a converged solution. A multitude of other applications, e.g. the detailed investigation of areas with pits and jets and thermal cracking is conceivable. Where the 1D formulation cannot be applied, the model may still serve to produce optimized input conditions for a $3 \mathrm{D}$ simulation to reduce computation time for an even more elaborate computation. While this can be expected to shorten the simulation time considerably, it still has to be checked which safe margin to apply to obtain a converged solution. As has been demonstrated in the current paper, even with optimized starting conditions, more than a couple of body rotations are typically necessary, especially in the case of unfavourable illumination conditions.

The method described in this paper has been validated against an application involving spacecraft data in reproducing temperature curves derived from fluxes measured by a TM onboard lander Philae within the limits of measurement errors and the assumption of a constant thermal inertia. Furthermore, results have been compared to those from independently developed 3D TPMs for a simplified shape model. A further model verification and application is currently underway for observations of Itokawa, taking into account unresolved small-scale surface roughness as well. In many cases, the effects of thermal inertia and surface roughness are entangled. Based on observation geometry and wavelength coverage, it is possible to constrain surface roughness (Delbo et al. 2015). O'Rourke et al. (2012) show this for a TPM fit to asteroid Lutetia. This case therefore is considered a very suitable test for the current method to be carried out in the future.

\section{ACKNOWLEDGEMENTS}

The author acknowledges funding by the Initiative and Networking Fund of the Helmholtz Association through the Helmholtz Postdoc Programme. This project has received partial funding from the $\mathrm{Eu}-$ ropean Union's Horizon 2020 research and innovation programme under grant agreement No 686709. The author thanks Line Drube for brainstorming on the Itokawa example and the rough properties model with region-specific thermal inertia, and Ekkehard Kührt for valuable discussions on model implementation.

\section{REFERENCES}

Biele J. et al., 2015a, in TherMoPHS II, http://elib.dlr.de/96227/

Biele J. et al., 2015b, Science, 349

Capanna C., Jorda L., Lamy P., Gesquière G., Delmas C., Durand J., Gaudon P., Jurado E., 2015, in AAS/Division for Planetary Sciences Meeting Abstracts. p. 413.11

Capria M. T. et al., 2017, MNRAS, 469, S685

Davidsson B. J., Rickman H., 2014, Icarus, 243, 58

Davidsson B. J. R. et al., 2015, Icarus, 252, 1

Delbo M., Mueller M., Emery J. P., Rozitis B., Capria M. T., 2015, Asteroid Thermophysical Modelling, University of Arizona Press, Tucson, Arizona. p. 107

Demura H. et al., 2006, Science, 312, 1347

Gaskell R. et al., 2008, Gaskell Itokawa Shape Model V1.0. HAY-AAMICA-5-ITOKAWASHAPE-V1.0. NASA Planetary Data System

Gulkis S. et al., 2012, Planet. Space Sci., 66, 31

Gulkis S. et al., 2015, Science, 347, aaa0709

Gundlach B., Blum J., 2012, Icarus, 219, 618

Gundlach B., Skorov Y., Blum J., 2011, Icarus, 213, 710

Harris A. W., Drube L., 2016, ApJ, 832, 127

Keihm S. et al., 2012, Icarus, 221, 395

Keller H. U. et al., 2015, A\&A, 583, A34

Kömle N. I. et al., 2017, MNRAS, 469, S2

Kuehrt E., 1984, Icarus, 60, 512

Lagerros J. S. V., 1996, A\&A, 310, 1011

Lagerros J. S. V., 1997, A\&A, 325, 1226

Lagerros J. S. V., 1998, A\&A, 332, 1123

Marsset M. et al., 2017, A\&A, 604, A64

McKay C. P., Squyres S. W., Reynolds R. T., 1986, Icarus, 66, 625

Miyamoto H. et al., 2007, Science, 316, 1011

Mottola S. et al., 2014, A\&A, 569, L2

Müller T. G., Hasegawa S., Usui F., 2014, PASJ, 66, 52

Müller T. G. et al., 2017, A\&A, 599, A103

O'Rourke L. et al., 2012, Planet. Space Sci., 66, 192

Pelivan I., Drube L., Kührt E., Helbert J., Biele J., Maibaum M., Cozzoni B., Lommatsch V., 2017, Adv. Space Res., 59, 1936

Preusker F. et al., 2015, A\&A, 583, A33

Preusker F. et al., 2017, A\&A, 607, L1

Rozitis B., Green S. F., 2011, MNRAS, 415, 2042

Schloerb F. P. et al., 2015, A\&A, 583, A29 
Seiferlin K., Kömle N., Kargl G., Spohn T., 1996, Planet. Space Sci., 44, 691

Spohn T. et al., 2015, Science, 349, aab0464

Steiner G., Kömle N. I., Kührt E., 1991, Theoretical Modelling of Comet Simulation Experiments, VÖAW , Graz. p. 11

Takita J., Senshu H., Tanaka S., 2017, Space Sci. Rev., 208, 287
Ulamec S. et al., 2016, Acta Astronaut., 125, 80

Wesselink A. J., 1948, Bull. Astron. Inst. Neth., 10, 351

This paper has been typeset from a TEX/LATEX file prepared by the author. 